\title{
Wind in the WiPPL
}

Nat. Phys. https://doi.org/10.1038/s41567-019-0592-7 (2019)

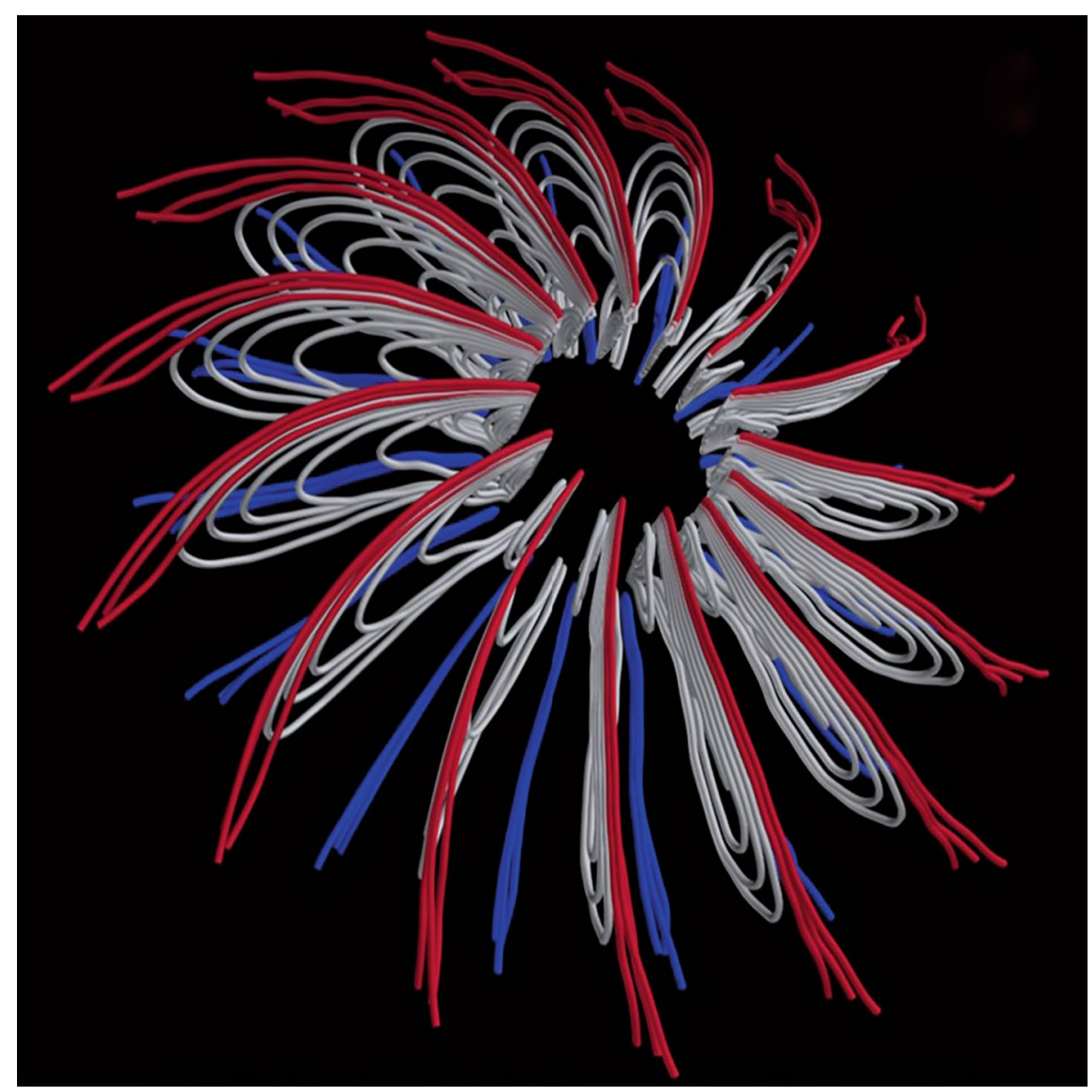

Credit: Springer Nature Ltd

As the Sun rotates, its magnetic field interacts with the solar wind and gets twisted into a spiral shape known as the Parker spiral (after Eugene Parker), the largest magnetic structure in the heliosphere. To get to grips with the dynamics of a rapidly rotating magnetosphere, Ethan Peterson and co-workers recreate the Parker spiral in the laboratory, where they can control the magnetic field strength, plasma density and temperature, ion mass and local solar wind speed.

Using the Big Red Ball facility at the Wisconsin Plasma Physics Laboratory (WiPPL), a 3-metre-diameter device for confining unmagnetized plasmas, the authors use a dipole magnet plus electromagnetic stirring techniques to effect a rotating stellar wind. At the point where the centrifugal force of the wind overcomes the magnetic confining force (the Alfvén speed), they observed the plasma breaking free from the magnetosphere and twisting the magnetic field into a Parker spiral. This transition produces a dynamic interface separating the closed magnetic field lines (pictured as white loops) of the magnetosphere and open magnetic field lines (pictured as blue and red lines) of the Parker spiral, at which crossed current and magnetic field lines give rise to the Hall effect that pushes ions inward (and electrons outward). The accumulating ions cause the closed magnetic field lines to bulge outward, until they reconnect to eject blobs of plasma (plasmoids) into the outflow at super Alfvénic speeds. Such plasmoids are observed in coronal 'helmet streamers'. Simulations without the Hall effect produce no plasmoids.

Insights into the structure and dynamics of a laboratory solar wind will complement the measurements of the Parker Solar Probe.

\section{May Chiao}

Published online: 28 August 2019 https://doi.org/10.1038/s41550-019-0895-8 